Centre for Academic Primary Care, Bristol Medical School, University of Bristol, Bristol, UK

2 Institute of Immunology and Immunotherapy, University of Birmingham, Birmingham, UK

3 Test Evaluation Research Group, Institute of Applied Health Research, University of Birmingham, Birmingham, UK

4 NIHR Birmingham Biomedical Research Centre at the University Hospitals Birmingham NHS Foundation Trust and the University of Birmingham, UK

Correspondence to J Watson Jessica.Watson@bristol.ac.uk Cite this as: BMJ2020;370:m3325 http://dx.doi.org/10.1136/bmj.m3325 Published: 08 September 2020

PRACTICE POINTER

\title{
Testing for SARS-CoV-2 antibodies
}

\author{
Jessica Watson, ${ }^{1}$ Alex Richter, ${ }^{2}$ Jonathan Deeks ${ }^{3,4}$
}

What you need to know

- Positive antibodies show evidence of previous exposure to SARS-CoV-2 virus

- Antibody testing should be undertaken at least two weeks after onset of symptoms

- The sensitivity and specificity of antibody tests vary over time and results should be interpreted in the context of clinical history

- Antibody testing might have a useful role in diagnosing covid-19 in patients with late presentation, prolonged symptoms, or negative results from reverse transcription polymerase chain reaction tests

- Evidence is currently insufficient to know whether individuals with SARS-CoV-2 antibodies have protective immunity

As the covid-19 pandemic has unfolded, interest has grown in antibody testing as a way to measure how far the infection has spread and to identify individuals who may be immune. ${ }^{1}$ Testing also has a clinical role, given the varying symptoms of covid-19 and false negative results of reverse transcription polymerase chain reaction (RT-PCR) tests, particularly when swabs are taken more than five days after symptom onset and sensitivity of RT-PCR tests starts to decrease. ${ }^{23}$ In May, the UK government announced that antibody testing should be offered to anyone having their blood taken who wants to know whether they have been infected with SARS-CoV-2, even if there is "not a specific clinical indication," 4 yet currently there is no clear guidance for clinicians on how to interpret these results or how they fit into clinical pathways. In this article we offer an approach to antibody testing in individuals with and without symptoms suggestive of current or past SARS-CoV-2 infection.

\section{How might antibody testing be used?}

Covid-19 antibody testing has been the focus of much research and press coverage. Four possible reasons are proposed for SARS-CoV-2 antibody testing:

- For diagnosis of individuals with current symptoms suggestive of covid-19, when antigen testing has failed to detect SARS-CoV-2, especially in those who present two weeks or more after symptom onset (when antibody testing becomes more reliable).

- For individuals who are currently asymptomatic, to assess if they have had a previous SARS-CoV-2 infection. This may include people at high risk of severe disease or those with occupational risk of infection (eg, healthcare workers) to provide reassurance, or to inform personal decisions about returning to work.
- To monitor the quality and longevity of the immune response in patients with previously confirmed covid-19 disease or potentially to monitor response to vaccination. If treatment with convalescent plasma is found effective in treating covid-19, antibody tests will also have a role in identifying suitable donors.

- For seroprevalence surveys for research and public health monitoring.

\section{What antibody tests are available?}

Three main types of antibody are produced in response to infection; IgA, IgG, and IgM. IgM rises soonest and typically declines after infection. IgG and IgA persist and usually reflect longer term immune response. Antibody tests look for a variation in the above antibodies, either as a separate or combined antibody measurement. Antibody tests can be done in laboratory settings using enzyme linked immunosorbent assays or chemiluminescence immunoassays (CLIA) usually using venous blood samples. Point of care tests that use disposable devices called lateral flow assays of finger prick blood are also available (including the UK-Rapid Test Consortium "AbC-19TM Rapid Test" which may soon be widely available to the public). The main tests currently used in the UK are the Abbott SARS-CoV-2 assay, which detects IgG, and the Roche Elecsys assay, which detects both IgM and IgG. Both are CLIA assays which require venous blood.

\section{Accuracy of antibody tests}

Accuracy is a measure of how well the tests detect previous SARS-CoV-2 infections, and not a direct measure of immunity to future infections. The accuracy of SARS-CoV-2 antibody tests is measured by comparing the test results with a gold standard: usually viral RNA detection by PCR testing at the time of symptoms. A limitation of this approach is the sensitivity in PCR testing (which may be as low as $70 \%)^{2}$

A Cochrane review of SARS-CoV-2 antibody testing included 57 publications on 54 cohort studies with 15976 samples, of which 8526 were from cases of confirmed SARS-CoV-2 infection. ${ }^{5}$ Measures of diagnostic accuracy varied depending on the timing of the tests (table 1). The maximum sensitivity for combined IgG or IgM tests was 96\% at days 22-35 after symptom onset. For IgG alone the maximum sensitivity was $88.2 \%$ at days $15-21$ after symptom onset. Summary specificities were provided in 35 out of 54 studies and exceeded $98 \%$ for all types of antibody test. 


\begin{tabular}{|c|c|c|c|c|c|c|}
\hline & Sensitivity & & & & & Specificity \\
\hline & Days 1-7 & Days 8-14 & Days 15-21 & Days 22-35 & Days >35 & All time points \\
\hline $\lg G^{*}$ & $29.7 \%(22.1-38.6)$ & $66.5 \%(57.9-74.2)$ & $88.2 \%(83.5-91.8)$ & $80.3 \%(72.4-86.4)$ & $86.7 \%(79.6-91.7)$ & $99.1 \%(98.3-99.6)$ \\
\hline $\lg M$ & $23.2 \%(14.9-34.2)$ & $58.4 \%(45.5-70.3)$ & $75.4 \%$ (64.3-83.8) & $68.1 \%$ (55.0-78.9) & $53.9 \%(38.4-68.6)$ & $98.7 \%(97.4-99.3)$ \\
\hline $\lg A$ & $28.4 \%(0.9-94.3)$ & $78.1 \%(9.5-99.2)$ & $98.7 \%(39.0-100)$ & 98.7\% (91.9-99.8) & $100 \%(85.2-100)$ & \\
\hline
\end{tabular}

These estimates of accuracy should be interpreted with caution. Of studies in the review, $89 \%$ were judged to be at high risk of bias, with the potential consequence that many of the tests are likely to be less sensitive than reported (meaning increased likelihood of false negatives). Most studies in the review only included patients who were diagnosed based on a positive RT-PCR test, which means that patients who have signs, symptoms, and exposure to SARS-CoV-2 but negative PCR (who are defined in the China Center for Disease Control and Prevention and World Health Organization case definitions as "probably covid") are excluded. This is important, as false negative rates of RT-PCR have been estimated between $2 \%$ and $29 \% .{ }^{6}$ Most studies recruited patients who were in hospital, who often had severe symptoms, and who are likely to have a greater antibody response than those in community settings. None directly measured test accuracy in asymptomatic patients, who have been shown to have lower levels of IgG and greater reductions in antibody levels in the early phase of infection. ${ }^{7}$ Nearly all studies sampled covid-19 cases and non-cases separately; this methodology leads to bias and tends to overestimate measures of accuracy. ${ }^{8}$ Data on accuracy of tests beyond 35 days was lacking. Tests performed after five weeks should be interpreted with additional caution, as some evidence suggests that antibody levels may wane, which would reduce an antibody test's sensitivity further. ${ }^{9}$

\section{Interpreting antibody tests}

Interpretation of test results depends not only on the accuracy of the test itself but also the pre-test probability of infection. This will vary widely depending on the indication for testing: when screening asymptomatic individuals the pre-test probability will be relatively low, for those with suggestive symptoms it is likely to be much higher. We illustrate this with two (fictitious) clinical cases.

\section{Case 1}

Anthony is 53, has type 2 diabetes, and a raised body mass index. He works as a security guard in a shopping centre in Norwich. His wife is worried about his risk of exposure to covid-19 at work, and phones the GP surgery requesting an antibody test. He has not had any suggestive symptoms and has no known exposure.

Anthony's pre-test probability can be estimated based on the population SARS-CoV-2 antibody seroprevalence in his area; in the East of England this is estimated to be around $10 \% .{ }^{10}$ As he has had no symptoms or known exposure his probability of asymptomatic seroconversion is likely to be lower; for illustrative purposes we estimate his pre-test probability at $5 \%$.

We do not have any data on the accuracy of antibody assays in asymptomatic people on which to base our estimates. We will start by using the average sensitivity of $91.4 \%$ and average specificity of $98.7 \%$ from the Cochrane review and consider what would change if, as is likely, the test had a lower sensitivity. Figure 1 illustrates the outcomes of testing based on 1000 people like Anthony, with a pre-test probability of $5 \%$. We would expect that 942 people would test negative, of whom four (0.4\%) would actually have had covid-19 (false negatives). Considering that the test may well have a lower sensitivity, particularly if the peak incidence and therefore likely time of infection is $>35$ days ago, this would proportionally increase the false negative rate. If the test made five times as many false negatives (sensitivity of $57 \%$ ) then this would rise to 20 false negatives (2.1\%)-still relatively low numbers owing to the low prevalence. A negative test result would therefore mean Anthony is unlikely to have had covid-19 infection. However, of the 58 people who would test positive, 12 people $(21 \%)$ would be falsely positive. This is important because a false positive could potentially influence Anthony's behaviour and adherence to infection control measures. This could be particularly risky as Anthony has an occupational risk of exposure and comorbidities, placing him at higher risk of complications from covid-19. The GP should therefore explain that the test result cannot be used to indicate immunity, and that regardless of the results of testing, Anthony should follow recommended precautions to avoid exposure to SARS-CoV-2. The test result in this case is therefore unlikely to change any advice given to the patient, and has the potential to cause harm through false reassurance. 


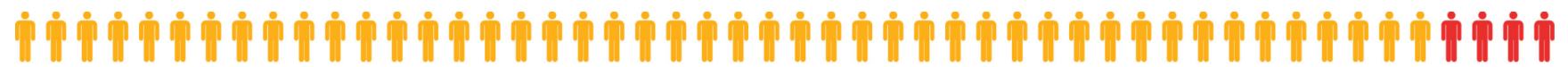

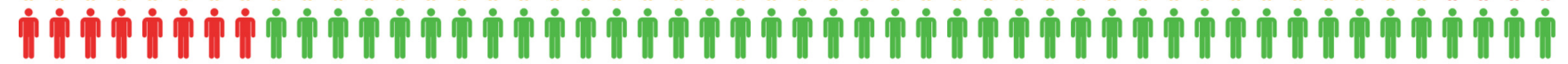

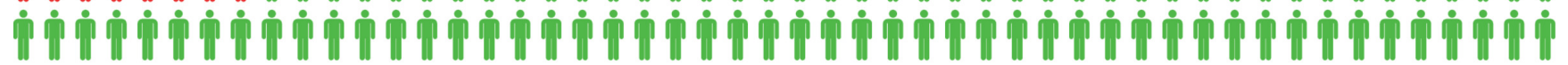

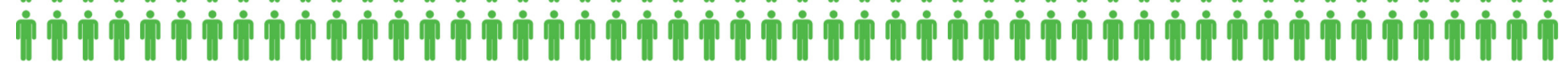

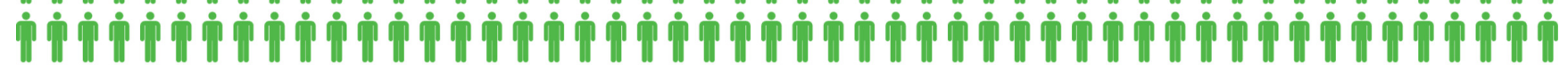

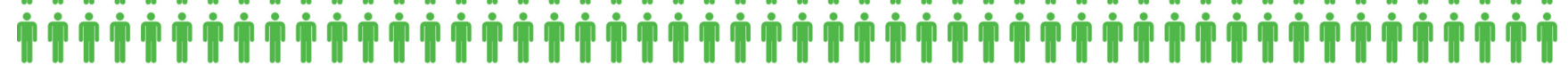

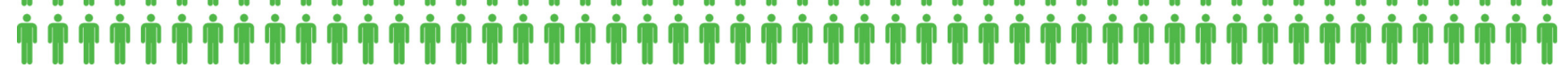

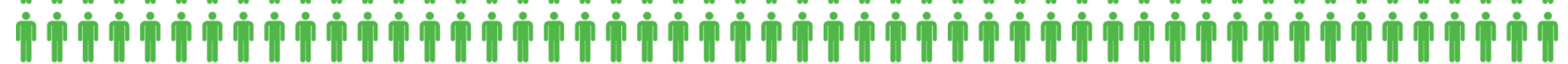

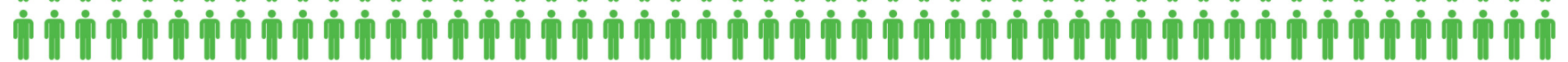

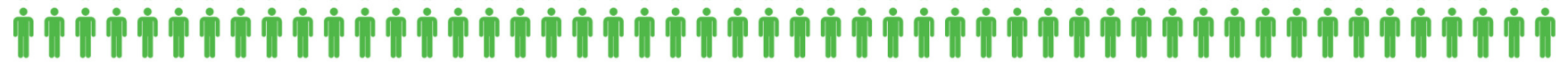

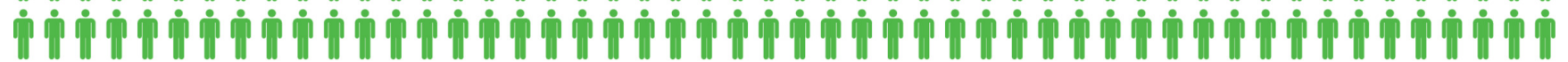

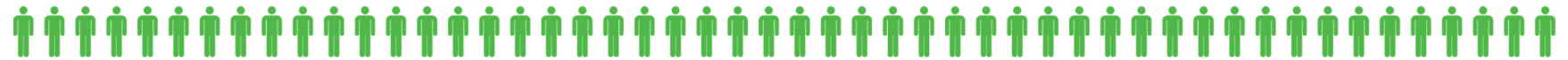

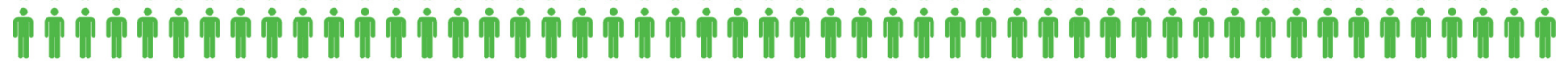

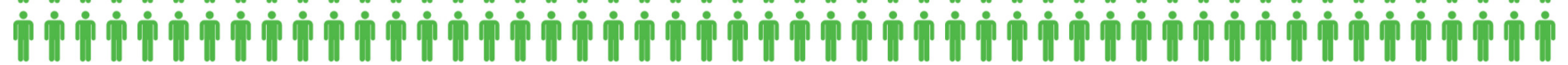

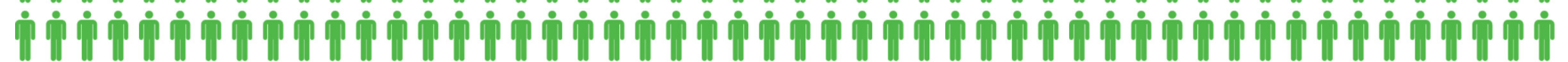

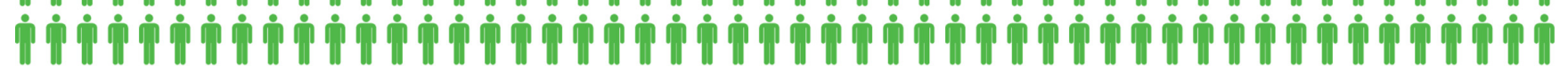

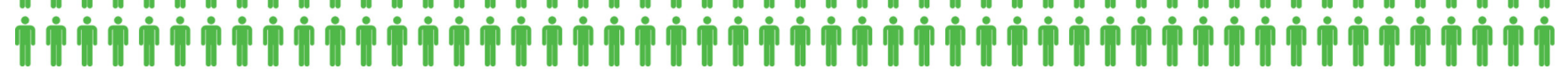

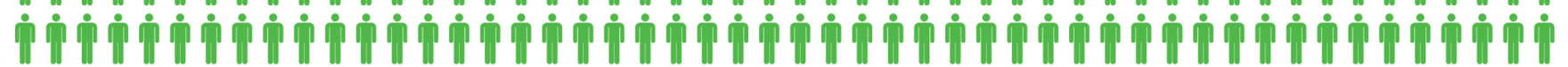

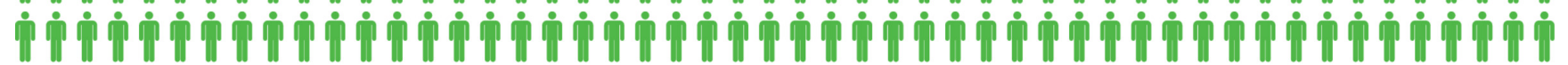

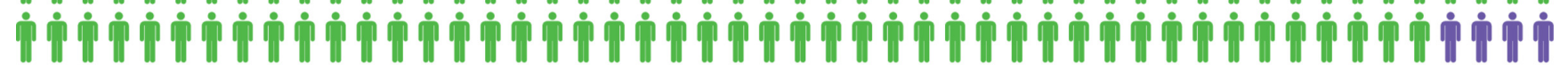

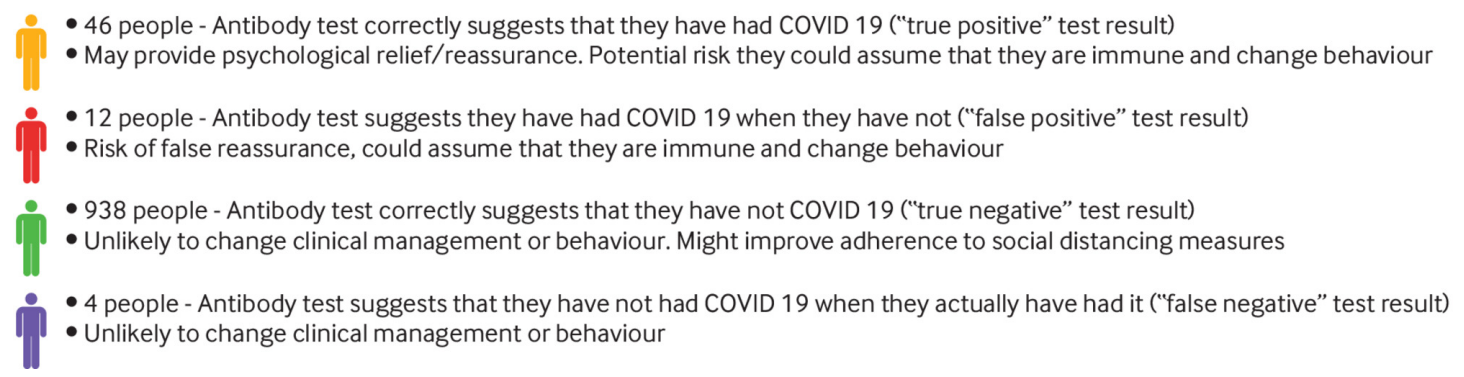

Fig 1 | Infographic showing outcomes of SARS-CoV-2 antibody testing based on 1000 people with a pre-test probability of $5 \%$

\section{Case 2}

Sarah is 32 and has been unwell for four weeks with intermittent shortness of breath, myalgia, atypical chest pains, fatigue, and anosmia. She never received a covid-19 swab test, as she did not have typical cough or fever symptoms.

Sarah has prolonged symptoms which are in keeping with a possible diagnosis of covid-19, although she has not had the cardinal features of cough or fever. To clarify the underlying cause of Sarah's symptoms before embarking on further investigations, her GP requests blood tests including covid-19 antibodies. Her pre-test probability will be higher than for Anthony, and will also depend on where she lives and whether she is known to have been exposed to the virus-for illustrative purposes we will estimate her pre-test probability at $50 \%$. We will use the estimates of sensitivity and specificity for the test from the Cochrane review. ${ }^{5}$

Figure 2 shows the outcomes of testing based on 1000 people with a pre-test probability of 50\%; 537 people would be expected to test negative, of whom 43 (8\%) would have actually had covid-19 (false negatives). If the sensitivity was not as high as in the Cochrane review (which is likely because of the limitations of the primary studies as discussed above) the number of false negatives would increase. This means a negative test in a patient like Sarah makes covid-19 less likely, but does not rule it out; Sarah might have had covid-19 but never developed an antibody response, her antibody levels could have dropped in the four weeks since symptom onset, or the test might have been unable to detect the antibodies that were present. However, the negative result would alert the clinician 
to consider other possible causes for Sarah's symptoms, which could help prevent missed or delayed diagnosis of other diseases in patients with symptoms assumed to be covid-19 related.

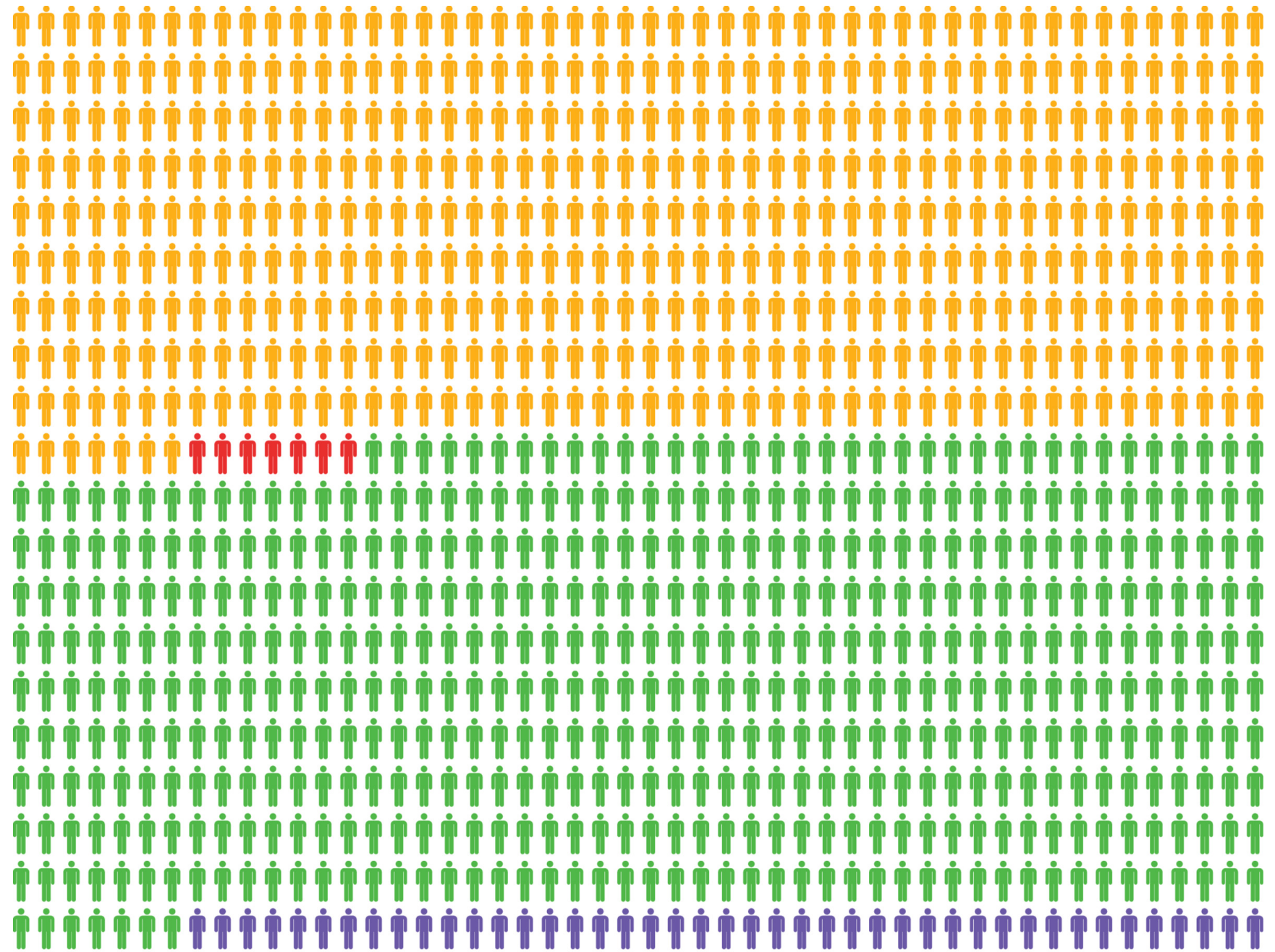

- 457 people - Antibody test correctly suggests that they have had COVID 19 ("true positive" test result)

- May provide relief/reassurance and help guide management of ongoing symptoms. Potential risk they assume that they are immune and change behaviour

$\bullet 7$ people - Antibody test suggests they have had COVID 19 when they have not ("false positive" test result)

- Risk of false reassurance that symptoms are due to covid19 which could delay further investigation or diagnosis. Could assume that they are immune and change behaviour

- 494 people - Antibody test correctly suggests that they have not COVID 19 ("true negative" test result)

- Clinician likely to consider other possible causes for ongoing symptoms, may help prevent missed or delayed diagnosis of other diseases

- 43 people - Antibody test suggests that they do not COVID 19 when they actually do have it ("false negative" test result)

- Risk of increased anxiety and uncertainty about possible causes for ongoing symptoms. Clinician might over investigate looking for other diseases

Fig 2 | Infographic showing outcomes of SARS-CoV-2 antibody testing based on 1000 people with a pre-test probability of $50 \%$

A positive test in this context would be much more compelling; of 1000 people tested, 464 people would test positive and only seven (2\%) would not have covid-19 (false positives). A positive test result in the context of suggestive symptoms therefore makes covid-19 infection highly probable (but doesn't exclude dual pathology). Antibody testing is therefore likely to be helpful in guiding clinical management of symptomatic patients like Sarah.

In summary, antibody tests have a high specificity, but sensitivity is variable and depends on time since symptom onset. Negative results should therefore be interpreted with caution in the context of typical symptoms. High specificity means false positives are uncommon ( $<2 \%$ of people who have not had covid-19 will have a false positive test). However in low prevalence settings true positives are also uncommon, which means the predictive value of a positive test will be lower in individuals with a low background risk of infection. Interpret a positive test in a patient with a low pre-test probability with caution, as false positives could lead to false reassurance with potential for patient harms. The interactive calculator on BMJ.com (https://sandpit.bmj.com/graphics/2020/c19testA/) allows clinicians to explore the impact of changing the pre-test probability, sensitivity, and specificity on test outcomes. Measures to reduce transmission, such as social 
distancing, should be maintained regardless of test result and we do not recommend antibody testing for this purpose at present.

\section{SARS-CoV-2 antibody tests and immunity}

Antibodies are an essential component of the adaptive immune response, providing specificity and memory against future infection. This is achieved through neutralisation by binding pathogens, activation of complement to destroy cells by lysis, presentation or opsonisation to immune cells to facilitate phagocytosis,

degranulation, and antibody dependent cell mediated cytotoxicity.

However, for many viruses and intracellular infections such as tuberculosis, T cell immunity is predominant. The role of T cells following SARS-CoV-2 infection is widely discussed..$^{11} \mathrm{~T}$ cell memory has been demonstrated in laboratory tests, and cross reactivity of $\mathrm{T}$ cell responses to other coronavirus infections potentially explains some of the variation in clinical severity of infection. ${ }^{12}$ As for most intracellular infections, it is likely that a combination of $\mathrm{B}$ and $\mathrm{T}$ cell immunity is involved in clearing covid-19 infection and generating protective memory.

Although we can test for the presence of antibodies, the extent to which SARS-CoV-2 antibodies provide future immunity and protection from repeat infection is not yet known. Experimental evidence shows neutralisation with certain SARS-CoV-2 antibodies, and inferred clinical evidence from very few reports shows repeat infection and successful use of convalescent plasma therapy. ${ }^{13} 14$ However, longitudinal studies are now reporting and showing that antibody levels are waning, ${ }^{9}$ and whether protective immunity will be maintained with a lower antibody titre is unknown.

To know whether our current antibody tests are indicative of protective immunity, ideally we would need disease prevalence studies in individuals with known antibody status; however, knowing whether these antibodies are neutralising in a laboratory should give us some indication before large population studies can be completed.

Lastly, antibodies have the ability to provide long term immunity but non-neutralising antibodies can also be produced, and a phenomenon known as antibody enhancement can occur where antibodies facilitate a secondary infection that can be more severe than the primary infection. ${ }^{15}$ This has been reported with other ${ }^{15}$ coronaviruses, but not to date with SARS-CoV-2.

\section{Pitfalls of antibody testing}

Policies on testing that are population based and without a specific clinical indication essentially amount to screening. This risks potential harm if the consequences of testing are not carefully considered. If testing is based on patient request, rather than clinically driven, we anticipate that rates of testing will be higher in more affluent populations, who are at lower risk of covid-19, in keeping with the inverse care law. ${ }^{16}$ This also limits the usefulness of data for estimates of seroprevalence, as a self-selecting population will not be representative. Concerns have been raised about the implications of the rapid rollout of antibody testing ${ }^{17}$ and the chief medical officer in Scotland has advised against on-demand testing. ${ }^{18}$

The Royal College of Pathologists has produced a covid-19 testing strategy, underpinned by seven principles, one of which is that testing must be carried out for a purpose. ${ }^{19}$ It is arguable that doctors commonly use tests for the purposes of reassurance, ${ }^{20}$ and this is therefore a justifiable rationale for testing. However, we would caution against requesting SARS-CoV-2 antibody tests for reassurance; two systematic reviews of randomised controlled trials found no evidence of effect of diagnostic tests on illness worry, non-specific anxiety, or symptom persistence ${ }^{21} 22$ and we do not know the effect of covid-19 testing on patient anxiety. Some suggest that the purpose of antibody testing should be to guide re-opening of workplaces ${ }^{23}$; however, until more is known about the relation between antibodies and protective immunity, results should not influence public health advice to individuals or workplaces. Even if future evidence shows that antibodies do confer sufficient and lasting immunity, the concept of "immunity passports" raises ethical issues, threatening freedom and fairness and potentially risking public health by incentivising people to wilfully seek out infection and antibody testing or encouraging a potential antibody testing black market. ${ }^{24}$

\section{Communicating risk and uncertainty}

Covid-19 offers an opportunity to improve clinician and patient understanding and communication of risk and uncertainty in diagnostic testing. Residual uncertainty after diagnostic testing is normal, and the same principles discussed here apply to most screening and diagnostic tests. We hope that the interest in covid-19 testing will lead to a wider debate around testing and test evaluation where accuracy of tests haven't been as closely scrutinised. Clinicians should consider the risks and benefits of SARS-CoV-2 antibody testing for individuals, and share information about the limitations of testing with patients (box 1). High quality evidence on test accuracy is currently lacking, and further research is needed to address areas of uncertainty (box 2). A drive to increase volumes of tests performed without considering the clinical value of testing could be an expensive distraction from key public health interventions. Yet carefully considered testing, in patients with late presentation of the illness, or prolonged or atypical symptoms could help reduce uncertainty, guide ongoing management, and improve understanding of the late sequelae of covid-19.

\section{Box 1: What you might tell your patient}

- Antibody tests help us find out who has had covid-19 in the past

- They cannot tell us for sure whether you can catch covid-19 in the future

- If the test is positive then it is likely that you have been infected at some time

- A negative test result cannot rule out the possibility that you have had covid-19

\section{Box 2: Uncertainties}

- Most studies on antibody tests are from patients in hospital. We do not know how well the tests work in patients with mild illness who were not admitted to hospital, or in people who are asymptomatic

- Data are lacking on test accuracy beyond 35 days-we do not know how well these tests will work for infections that occurred more than five weeks ago

- Evidence is insufficient to know whether the presence of antibodies confers lasting immunity to protect against future covid-19 infection

\section{How patients were involved in the creation of this article}

Two patient representatives from the University of Birmingham patient and public involvement panel reviewed this article. The feedback was that the article was interesting and readable, and the case studies were realistic. As a result of the feedback, we made changes to the wording of box 1 , "What you might tell your patient." 


\section{How this article was made}

This article was produced at speed to address an urgent need for evidence. JD has recently led a Cochrane systematic review of the diagnostic accuracy of covid-19 antibody tests, and this paper is based on the evidence from this systematic review, with clinical input from JW and AR.

\section{Education into practice}

- What is the protocol for SARS-CoV-2 antibody testing in your organisation?

- How do you explain SARS-CoV-2 antibody test results to patients?

- Reflect on a recent case of SARS-CoV-2 antibody testing-did the test results influence clinical management?

Competing interests: We have read and understood the BMJ policy on declaration of interests and declare the following interests: AR is a collaborator from the University of Birmingham/Binding Site group developing assays for covid-19 antibody testing, JW and JD have no competing interests.

Funding: JW is funded by a doctoral research fellowship from the National Institute for Health Research (DRF-2016-09-034). JD is a UK National Institute for Health Research (NIHR) senior investigator emeritus and is supported by the NIHR Birmingham Biomedical Research Centre. This paper presents independent research supported by the NIHR Birmingham Biomedical Research Centre at the University Hospitals Birmingham NHS Foundation Trust and the University of Birmingham. The views expressed in this publication are those of the authors and not necessarily those of the NHS, the National Institute for Health Research, Health Education England, or the Department of Health.

Patient consent: The cases in this article are fictitious and therefore no consent was needed.

Provenance and peer review: commissioned, externally peer reviewed.

1 Petherick A. Developing antibody tests for SARS-CoV-2. Lancet 2020;395:1101-2.

2 Watson J, Whiting PF, Brush JE. Interpreting a covid-19 test result[PubMed]. BMJ 2020;369:m1808.

3 Kucirka LM, Lauer SA, Laeyendecker O, Boon D, Lessler J. Variation in false-negative rate of reverse transcriptase polymerase chain reaction-based SARS-CoV-2 tests by time since exposure. Ann Intern Med 2020;173:262-7.

4 Lind S. GPs to provide covid antibody testing for patients who have bloods taken. Pulse; 2020. http://www.pulsetoday.co.uk/news/gps-to-provide-covid-antibody-testing-for-patients-whohave-bloods-taken/20040894.article.

5 Deeks J), Dinnes J, Takwoingi Y, etalCochrane COVID-19 Diagnostic Test Accuracy Group. Antibody tests for identification of current and past infection with SARS-CoV-2[PubMed]. Cochrane Database Syst Rev 2020;6:.

6 Arevalo-Rodriguez I, Buitrago-Garcia D, Simancas-Racines D, et al. False-negative results of initial RT-PCR assays for covid-19: a systematic review. MedRxiv [Preprint] 2020. doi: 10.1101/2020.04.16.20066787\%).</unknown>

7 Long Q-X, Tang X-J, Shi Q-L, et al. Clinical and immunological assessment of asymptomatic SARS-CoV-2 infections. Nat Med 2020;26:1200-4.

8 Whiting PF, Rutjes AWS, Westwood ME, Mallett S; QUADAS-2 Steering Group. A systematic review classifies sources of bias and variation in diagnostic test accuracy studies. J Clin Epidemiol 2013;66:1093-104

9 Ibarrondo FJ, Fulcher JA, Goodman-Meza D, etal. Rapid decay of anti-SARS-CoV-2 antibodies in persons with mild covid-19[PubMed]. N Engl/ Med 2020; doi: 10.1056/NEJMc2025179.

10 Public Health England. Sero-surveillance of covid-19. 2020. https://www.gov.uk/government/publications/national-covid-19-surveillance-reports/sero-surveillance-of-covid-19.

11 Gallais F, Velay A, Wendling M-J, et al. Intrafamilial exposure to SARS-CoV-2 induces cellular immune response without seroconversion. MedRxiv [Preprint] 2020. doi: 10.1101/2020.06.21.20132449\%

12 Le Bert N, Tan AT, Kunasegaran K, et al. SARS-CoV-2-specific T cell immunity in cases of COVID-19 and SARS, and uninfected controls. Nature 2020;584:457-62.

13 Duan K, Liu B, Li C, et al. Effectiveness of convalescent plasma therapy in severe COVID-19 patients. Proc Natl Acad Sci U S A 2020;117:9490-6.

14 WuF, Wang A, Liu M, et al. Neutralizing antibody responses to SARS-CoV-2 in a covid-19 recovered patient cohort and their implications. MedRxiv [Preprint] 2020. doi: 10.1101/2020.03.30.20047365\%)

15 Fierz W, Walz B. Antibody dependent enhancement due to original antigenic sin and the development of SARS. Front Immunol 2020;11:1120.

16 Hart JT. The inverse care law. Lancet 1971;:1:405-12.

17 Andersson M, Low N, French N, et al. Rapid roll out of SARS-CoV-2 antibody testing-a concern. BMJ2020;369:m2420.

18 Scottish Government. COVID-19 antibody testing 2020. 2020 https://www.gov.scot/news/covid19-antibody-testing-1
19 Martin J. Covid-19 testing: a national strategy. Royal College of Pathologists 2020 https://www.rcpath.org/uploads/assets/2e8d8771-f85a-408a-b5c8e68969cd21d5/cbcb4f30d8f8-40fe-ba8cdef3e6803ee4/RCPath-COVID-19-testing-a-national-strategy.pdf

20 Watson J, de Salis I, Banks J, Salisbury C. What do tests do for doctors? A qualitative study of blood testing in UK primary care. Fam Pract2017;34:735-9.

21 Petrie KJ, Sherriff R. Normal diagnostic test results do not reassure patients. Evid Based Med 2014;19:14.

22 Rolfe A, Burton C. Reassurance after diagnostic testing with a low pretest probability of serious disease: systematic review and meta-analysis. JAMA Intern Med 2013;173:407-16.

23 Weinstein MC, Freedberg KA, Hyle EP, Paltiel AD. Waiting for certainty on covid-19 antibody tests -at what cost?[PubMed]. N Engl J Med 2020;383:. doi: 10.1056/NEJMp2017739.

24 Kofler N, Baylis F. Ten reasons why immunity passports are a bad idea. Nature 2020;581:379-81.

This article is made freely available for use in accordance with BMJ's website terms and conditions for the duration of the covid-19 pandemic or until otherwise determined by BMJ. You may use, download and print the article for any lawful, non-commercial purpose (including text and data mining) provided that all copyright notices and trade marks are retained. 